\title{
Mortality and implant revision rates of hip arthroplasty in patients with osteoarthritis: registry based cohort study
}

In the online version of this Research paper by D J W McMinn and colleagues (BMJ 2012;344:e3319, doi:10.1136/bmj.e3319) some late changes to the figures were not reflected in the

legends. In figures 1 and 2 the confidence intervals are not all shown as dashed lines, as stated in the legend, but as thinner versions of the lines showing the survival curves.

Cite this as: BMJ 2012;344:e4371

๑ BMJ Publishing Group Ltd 2012 